\title{
Síndrome metabólica em crianças e adolescentes: dúvidas na terminologia, mas não nos riscos cardiometabólicos
}

\author{
Metabolic syndrome in children and adolescents: doubts \\ about terminology but not about cardiometabolic risks
}

Durval Damiani', Valesca Mansur Kuba², Louise Cominato', Daniel Damiani', Vaê Dichtchekenian', Hamilton Cabral de Menezes Filho'

\begin{abstract}
1 Unidade de Endocrinologia Pediátrica, Instituto da Criança, Hospital das Clínicas, Faculdade de Medicina da Universidade de São Paulo (ICr-HC-FMUSP), São Paulo, SP, Brasil ${ }^{2}$ Instituto de Endocrinologia e Diabetes do Estado do Rio de Janeiro (IEDE); Pontifícia Universidade Católica do Rio de Janeiro (PUCRJ), Rio de Janeiro, RJ, Brasil ${ }^{3}$ Curso de Medicina, Universidade Cidade de São Paulo (Unicid) São Paulo, SP, Brasil
\end{abstract}

Correspondência para:

Durval Damiani

Rua Cônego Eugênio Leite, 693 ap. 151

05414-011 - Pinheiros, São Paulo, SP, Brasil

durvald@iconet.com.br

Recebido em 6/Out/2011 Aceito em 16/Out/2011

\section{SUMÁRIO}

A síndrome metabólica (SM) tem gerado enormes controvérsias, a iniciar-se pela terminologia. 0 próprio autor que sugeriu o termo, Gerald Reaven, recomenda que não o utilizemos, porque, como a síndrome é um apanhado de alterações metabólicas e três dessas alterações podem defini-la, fica sempre difícil saber-se a que conjunto de alterações estamos nos referindo quando conotamos o termo SM. Em crianças, a complicação é ainda maior, já que se fazem diferentes adaptações aos critérios propostos para adultos. De qualquer forma, independentemente da terminologia, os riscos cardiovasculares são bem estabelecidos e fica cada vez mais claro que as crianças, já em tenra idade, podem começar a ter alterações metabólicas preditivas de problemas mais sérios futuramente. Assim, o papel do endocrinologista pediátrico e do pediatra geral é de investigar essas crianças, especialmente se portadoras de sobrepeso ou obesidade, para detectar precocemente e intervir em condições que podem ser prevenidas na vida adulta. Essa é uma nova visão de uma abordagem pediátrica preventiva de problemas que, usualmente, só são diagnosticados em vida adulta. Nesta revisão, abordamos os aspectos de definição, epidemiologia, fisiopatologia e de complicações da SM em crianças e adolescentes. Arq Bras Endocrinol Metab. 2011;55(8):576-82

\section{Descritores}

Síndrome metabólica; dislipidemia; resistência à insulina; hipertensão arterial sistêmica; esteatose hepática; índice de massa corpóreo

\section{SUMMARY}

Metabolic syndrome (MS) has been a condition involved in considerable controversy, starting with the terminology. Gerald Reaven himself, the author who proposed the term MS, advised against the use of this terminology because the definition implies in at least three metabolic alterations, and it is never clear to which group of alterations we are referring to when we say that a patient has MS. In children, the problem is even more complicated, since there are many different adaptations to the criteria used in adults. On the other hand, independent of the terminology, cardiovascular risks are well-established and it is very clear that even children may present metabolic disturbances which predict future metabolic problems. The role of the pediatric endocrinologist or the general pediatrician is to investigate, especially in overweight/ obese children, conditions that if treated early, may prevent future complications that today, unfortunately, are being diagnosed only in adult life. In this review, we discuss problems on the definition, epidemiology, pathophysiology, and complications of MS in children and adolescents. Arq Bras Endocrinol Metab. 2011;55(8):576-82

\section{Keywords}

Metabolic syndrome; dyslipidemia; insulin resistance; systemic hypertension; hepatic steatosis; body mass index 


\section{INTRODUÇÃO}

$\mathrm{A}$ síndrome metabólica (SM) é definida como uma constelação de anomalias antropométricas, fisiológicas e bioquímicas que aumentam o risco de desenvolvimento de doença cardiovascular e diabetes melito do tipo 2 (DM2) (1). Inicialmente descrita por Kylin, em 1920, como a tríade hipertensão arterial sistêmica (HAS), hiperglicemia e gota, foi tendo seu nome e definição modificados, sendo renomeada por Reaven, em 1988, como síndrome X. Nesta última conceituação, a obesidade não fazia parte do diagnóstico. Kaplan, em 1989, chamou-a de "quarteto mortal" e atualmente é mais conhecida como "síndrome da resistência insulínica” ou "síndrome metabólica” (2).

Apesar de haver falta de padronização para a definição da SM, existem numerosos estudos que mostram que ela se desenvolve durante a infância, sendo muito prevalente entre crianças e adolescentes com sobrepeso ou obesidade (2). Um dado curioso é que a apneia obstrutiva do sono, que ocorre em $2 \%$ a $3 \%$ das crianças, e, mesmo o ronco primário, que ocorre em $3 \%$ a $12 \%$, podem já prenunciar o desenvolvimento de síndrome metabólica em época posterior da vida (3).

Em 2004, várias entidades tentaram uma padronização dos critérios diagnósticos, com o objetivo de se programarem estratégias terapêuticas com potencial redução de riscos cardiometabólicos $(4,5)$. Nesse consenso, objetivava-se um diagnóstico fácil, que não necessitasse de métodos sofisticados, nem sempre disponíveis em todos os centros. Dessa forma, a resistência à insulina, pelas dificuldades técnicas de sua obtenção confiável, não foi adotada e a medida da circunferência abdominal (CA) foi adotada. A CA é o melhor parâmetro clínico para se inferir o grau de adiposidade abdominal, porém os valores variam com a etnia. Alguns autores, como Fernandez e cols. (4) e Katzmarzyk (5), já publicaram dados de percentis de CA para crianças e adolescentes. Maffeis e cols. demonstraram que crianças com CA > p90\% são mais propensas a ter múltiplos fatores de risco do que aquelas com menores percentis (6).

\section{DEFINIÇÃO}

O diagnóstico de SM no adulto é realizado quando há obesidade central e dois dos quatro critérios listados a seguir (7): CA > $90 \mathrm{~cm}$ em homens e $80 \mathrm{~cm}$ em mulheres sul-americanos/africanos; triglicérides $>150 \mathrm{mg} /$ $\mathrm{dL}$; HDL-colesterol $<40 \mathrm{mg} / \mathrm{dL}$ em homens e $<50$
mg/dL em mulheres; PA > 130/80 mmHg; glicemia de jejum alterada: $>100 \mathrm{mg} / \mathrm{dL}$.

A definição da SM na infância e na adolescência é problemática, já que pressão arterial (PA), perfil lipídico e valores antropométricos variam com a idade e o estádio puberal. Devem-se usar, portanto, diferentes pontos de corte para sexo e idade para cada uma das variáveis.

A Federação Internacional de Diabetes (IDF) definiu critérios de SM para crianças e adolescentes, dividindo-os em faixas etárias: 6 a $<10$ anos; 10 a $<16$ anos; > 16 anos.

Nesses três grupos, a CA > p90 para idade é imprescindível para o diagnóstico da síndrome. Abaixo de 10 anos, o diagnóstico de SM não deve ser feito, porém a criança deve ser orientada quanto à necessidade de perda de peso e mudança de estilo de vida. Acima de 10 anos, esse diagnóstico já pode ser realizado e, para isso, é necessário que haja obesidade abdominal e presença de dois ou mais dos seguintes fatores: triglicérides > $150 \mathrm{mg} / \mathrm{dL}, \mathrm{HDL}<40 \mathrm{mg} / \mathrm{dL}$, glicemia de jejum > $100 \mathrm{mg} / \mathrm{dL}$ e PA > p95. Para adolescentes maiores de 16 anos, são utilizados os critérios de adultos (8).

Acreditamos que um aspecto importante é não se ater a uma nomenclatura específica, no caso, SM, mas aos fatores de risco que podem estar presentes desde tenra idade. Os fatores individuais que compõem a SM agregam risco de doença cardiovascular, e a síndrome nada mais é do que a soma desses riscos. É por tal razão que alguns autores, incluindo o próprio Gerald Reaven, não aconselham mais o uso do termo SM, que acaba, no final das contas, a ser usado mais porque caiu no gosto do linguajar médico e talvez isso crie mais problemas do que resolva, já que, ao dizermos SM, não sabemos exatamente a que grupo de alterações aquela definição se refere.

A CA é considerada condição independente preditora de risco cardiovascular em adultos e crianças. É ainda um importante indicador de resistência insulínica, dislipidemia e hipertensão (os componentes da SM), além de se correlacionar com a proteína C-reativa (PCR), um marcador de processo inflamatório. Crianças com CA > p90 são mais propensas a múltiplos fatores de risco que crianças abaixo desse percentil (6).

Uma proposta interessante é a avaliação da relação entre a cintura abdominal (medida no ponto médio entre a última costela e a crista ilíaca ântero-superior) e a altura. Essa relação foi avaliada em mais de 3 mil crianças e adolescentes de 4 a 18 anos de idade, negros e brancos, encontrando-se um ponto de corte de 0,5 como 
indicativo de adiposidade visceral, mesmo em crianças com índice de massa corpórea normal (9). Num estudo realizado no Rio de Janeiro com 175 crianças de 6 a 10 anos por Kuba e cols. (comunicação pessoal), houve correlação significativa entre a relação CA/A e o IMC no que diz respeito ao diagnóstico nutricional de obesidade $9 \mathrm{R}=0,88, \mathrm{P}<0,0001)$. Quando o ponto de corte da CA/A foi igual a 0,51 , a sensibilidade para o diagnóstico de obesidade foi de $82,76 \%$ e a especificidade, de $97,73 \%$. Além disso, a CA/A apresentou correlação significante com alterações do HOMA-IR $(r=0,82$, $\mathrm{p}<0,0001)$, proteína $\mathrm{C}$ ultrassensível (PCR) $(\mathrm{r}=0,5 \mathrm{l}$, $\mathrm{p}<0,0001)$, pressão arterial e lípides.

\section{EPIDEMIOLOGIA}

A literatura mostra uma elevada correlação entre obesidade e prevalência de SM independentemente dos critérios utilizados. Esse fato, somado à epidemia atual de obesidade infantil, leva-nos a crer num desproporcional aumento de doenças cardiovasculares já na infância, além do desenvolvimento do DM2 e, consequentemente, da menor sobrevida da população.

Em menores de 10 anos, as alterações cardiometabólicas podem ser sutis, surgindo lenta e progressivamente. Por isso, é importante rastreá-las precocemente, em particular nas crianças com sobrepeso/obesidade, independentemente da história familiar de doença cardiovascular precoce e diabetes tipo 2 .

A obesidade e a SM estão aumentando em níveis epidêmicos, principalmente nas últimas três décadas, na população pediátrica. Nos Estados Unidos, comparando-se os inquéritos nacionais de 1985 e 1990, observou-se, em crianças de 6 a 11 anos, um aumento da obesidade de $67 \%$ nos meninos e de $42 \%$ nas meninas (10).

No Brasil, também vem ocorrendo um fenômeno de transição nutricional nas últimas décadas. O Instituto Brasileiro de Geografia e Estatística (IBGE) tem evidenciado uma queda apreciável na prevalência dos desnutridos e um aumento expressivo no excesso de peso, sendo que nas regiões Sudeste e Nordeste, em 10 anos, as taxas de sobrepeso aumentaram $0,5 \%$ ao ano, e a prevalência de sobrepeso/obesidade evoluiu de $4,15 \%$ para $13,9 \%(11)$.

Existem estudos em diferentes partes do mundo sobre prevalência de SM entre crianças e adolescentes. Esses números variam muito, já que diferentes critérios são utilizados. É sabido que a SM é mais prevalente entre crianças e adolescentes obesos (2).
Ferreira e cols. avaliaram 52 crianças brasilienses obesas entre 7 e 10 anos e constataram que 17,3\% apresentavam SM (12). Já no estudo de Bogalusa, a prevalência de SM foi de $3 \%$ em crianças negras e de $4 \%$ em crianças brancas. Esse estudo utilizou como critério de $S M$ a presença de quatro componentes acima do percentil 75 para sexo e idade (13). A prevalência encontrada pelo Third National Health and Nutrition Examination Survey (NHANES III) em adolescentes norte-americanos foi de 4,2\%. Nessa análise, a anormalidade mais comum foi hipertrigliceridemia e HDL baixo. Já a prevalência de glicemia de jejum alterada foi baixa $(1,5 \%)$. Nesse mesmo estudo, a prevalência de SM entre adolescentes foi de $28,7 \%, 6,1 \%$ e $0,1 \%$ para adolescentes obesos, sobrepeso e eutróficos, respectivamente. Esses resultados mostram, indubitavelmente, a influência da obesidade como fator etiológico independente para o desenvolvimento de doenças cardiovasculares e DM2 (7).

Kuba e cols. (comunicação pessoal) compararam a frequência de alterações cardiometabólicas entre 175 escolares de 6 a 10 anos (87 com excesso de peso) e observaram que, embora todas as alterações cardiometabólicas fossem mais frequentes no grupo com excesso de peso, a mais prevalente foi a hipertensão diastólica (72,7\% vs. 17\%).

No estudo do National Cholesterol Education que utilizou os critérios do Adult Treatment Panel III (ATP III) modificados para adolescentes, foram identificados 30\% de adolescentes com SM (14). Quando esses mesmos adolescentes foram submetidos a uma classificação de SM que levava em consideração o percentil de índice de massa corpórea (IMC), esse valor subiu para $31,3 \%$. Duncan e cols. estudaram 991 adolescentes entre 12 e 19 anos de idade usando os critérios modificados do ATP III e evidenciaram um aumento de SM de $4,2 \%$ para $6,4 \%$ em 10 anos (15). A prevalência de SM no estudo de 1.513 adolescentes brancos, negros e hispânicos feito por Goodman e cols. foi de 19,5\% pelos critérios do ATP III modificado, e 38,9\% pelos critérios da OMS $(16,17)$. Weiss e cols. revelaram taxas alarmantemente altas de SM entre jovens obesos: $38,7 \%$ em crianças moderadamente obesas, e $49,7 \%$ nas gravemente obesas (8). Esses autores usaram como critérios de SM: IMC > p97, triglicérides > p95, HDL $<$ p5 e glicemia entre 140 e $200 \mathrm{mg} / \mathrm{dL}$ no tempo de 120 minutos do teste de tolerância à glicose. Em nossos 220 adolescentes obesos acompanhados no Grupo de Obesidade do Instituto da Criança do Hospital das Clí- 
nicas da Faculdade de Medicina da Universidade de São Paulo (ICr-HC-FMUSP), encontramos a alarmante cifra de $71 \%$ de SM. Essas discrepâncias na prevalência de SM nos diferentes estudos ressaltam a necessidade de uma definição universal para a faixa etária pediátrica.

\section{ETIOLOGIA E FISIOPATOLOGIA}

A patogênese da SM é complexa e, até hoje, não foi totalmente elucidada. Existem dois fatores causais primordiais na gênese dessa doença: obesidade central e resistência à insulina. Outros fatores também são importantes: predisposição genética, inatividade física, alimentação inadequada, estado pró-inflamatório e alterações hormonais (18). Fatores pré-natais também são preditores da SM. Desse modo, crianças e adolescentes que foram pequenos para a idade gestacional (PIG) têm probabilidade aumentada de desenvolver alterações metabólicas, além de eventos cardiovasculares e DM2 na idade adulta (13).

Obesidade: o aumento do tecido adiposo é responsável por um estado pró-inflamatório, com aumento de liberação de citocinas (interleucinas e fator de necrose tumoral alfa) e diminuição de produção de adiponectina, resultando numa diminuição da sensibilidade à insulina. Substâncias como a omentina e a visfatina aumentam a sensibilidade à insulina, enquanto a resistina, também produzida pelo adipócito, aumenta a resistência à insulina.

Resistência insulínica: é um termo utilizado para descrever a capacidade diminuída dos tecidos-alvo (músculo, tecido adiposo e fígado) de responder à ação celular da insulina. A glicose passa a apresentar dificuldade para entrar nessas células, acumulando-se no sangue. Essa hiperglicemia desencadeia o aumento da produção pancreática de insulina, levando à hiperinsu- linemia compensatória, que mantém as concentrações glicêmicas dentro da normalidade. Quando as células betapancreáticas atingem seu limite secretor máximo e entram em falência, o paciente desenvolve DM2 (Figura 1). Mesmo antes de o DM2 ser diagnosticado, lesões microvasculares já estão ocorrendo e, desse modo, há comprometimento da retina, dos rins e dos nervos. $\mathrm{O}$ estudo de Bogalusa sugere que haja uma associação independente entre resistência à insulina, gordura corpórea e risco cardiovascular e, quando esses fatores são combinados, o aumento no risco cardiovascular é maior do que quando somente resistência à insulina ou gordura corpórea são avaliadas isoladamente, ou seja, há um efeito aditivo no risco (19). A inatividade física diminui as concentrações de GLUT-4, transportador que facilita a entrada de glicose nas células, e aumenta os níveis de ácidos graxos livres, já que aumenta a gordura visceral. Portanto, o sedentarismo também é importante causa de resistência insulínica (20).

Um ponto importante a ser discutido é como caracterizar resistência à insulina. O padrão-ouro (clamp euglicêmico hiperinsulinêmico) é impraticável na prática clínica, sendo restrito a trabalhos de investigação, já que é um procedimento complexo, difícil de ser realizado e composto de um número enorme de dosagens tanto de glicose quanto de insulina, por método que seja específico para a insulina (e não inclua, por exemplo, pró-insulina). Como alternativas, tem se usado o HOMA-IR (homeostasis model assessment - insulin resistance) em que se multiplicam as concentrações de glicose $(\mathrm{mg} /$ $\mathrm{dL}$ ) e insulina (mUI/L) e se divide o produto por 405 . Dependendo do ponto de corte utilizado, a prevalência de resistência à insulina varia de $45 \%$ a $80 \%$ em crianças obesas, $10 \%$ a $60 \%$ em sobrepeso e $2 \%$ a $20 \%$ em crianças de peso normal $(1,21)$.

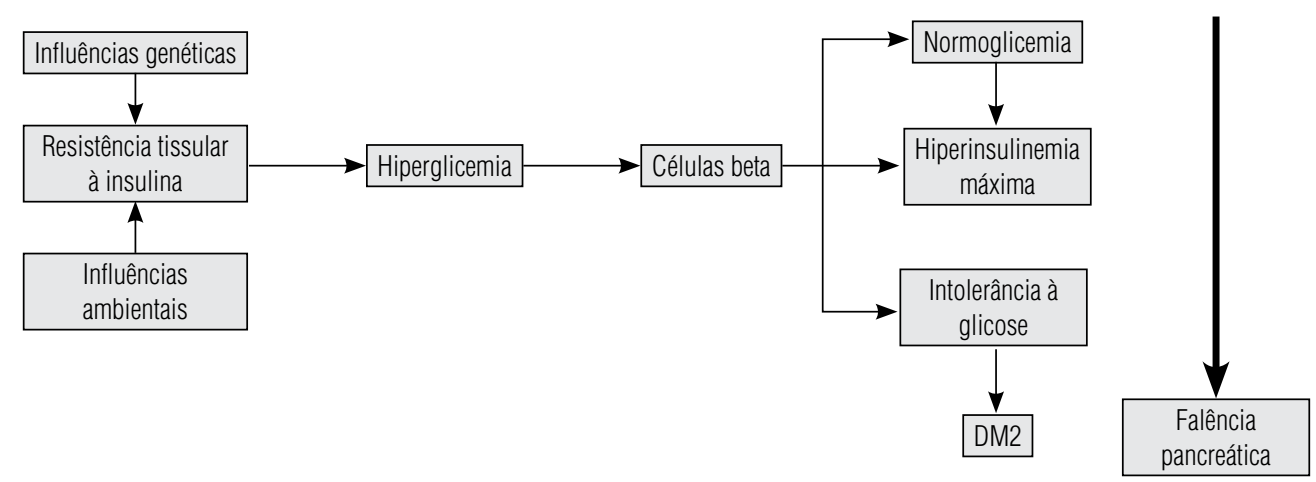

Figura 1. A incapacidade da célula beta em compensar a resistência à insulina determina 0 aparecimento do diabetes melito tipo 2 (DM2). 
Crianças e adolescentes que foram pequenas para a idade gestacional (PIG) têm risco aumentado de desenvolver SM. Os mecanismos que explicam essa relação ainda são obscuros. A hipótese mais aceita é a da programação fetal, na qual a adaptação fetal à exposição a poucos nutrientes leva a uma inadaptação à exposição de nutrientes de forma abundante (13). O risco de desenvolver SM é maior entre as crianças que apresentaram catch-up growth (crescimento compensatório) nos primeiros dois anos de vida (22).

Dietas ricas em carboidratos de fácil absorção, com alto teor lipídico, porém pobres em fibras, levam a ganho de peso e resistência insulínica, aumentando a probabilidade de desenvolver SM.

\section{COMPLICAÇÕES}

Atualmente são acompanhados, no Ambulatório de Obesidade do ICr-HC-FMUSP, 220 adolescentes obesos com IMC > p95, com idade de 10 a 19 anos, com média de IMC de 35,02+/-7,69.

Entre eles, 75,4\% apresentam resistência à insulina, $71 \%$ preenchem os critérios de síndrome metabólica, $50 \%$ apresentaram dislipidemia, 25\%, esteatose hepática e $22,3 \%$, hipertensão arterial. Glicemia de jejum alterada e DM2 ocorrem em 5\% e 2\%, respectivamente. Vamos abordar com mais detalhes algumas das complicações relacionadas à SM em crianças e adolescentes, tais como a dislipidemia, a hipertensão arterial sistêmica, o estado pró-trombótico, a doença cardiovascular, o DM2 e a esteatose hepática (23).

\section{Dislipidemia}

A resistência insulínica no tecido adiposo leva ao aumento de ácidos graxos livres. Essas substâncias aumentam a produção hepática de lipoproteína de baixa densidade (LDL), elevando as concentrações plasmáticas de triglicérides e apolipoproteína $\mathrm{B}$, além de diminuírem as concentrações de HDL. As partículas de LDL são mais suscetíveis à oxidação na parede da artéria, favorecendo a formação da placa aterosclerótica; em contrapartida, o HDL tem um papel cardioprotetor já bem estabelecido. A sua propriedade antiaterosclerótica ocorre pela sua habilidade em promover a saída do colesterol das células. Esse processo minimiza o acúmulo de células espumosas na parede arterial. Também funcionam como inibidores da oxidação do LDL-colesterol, ligando-se a potentes antioxidantes (chamados paraoxonases), e da expressão das moléculas de adesão na célula endotelial, evitando, assim, a aterogênese. Estudos cinéticos em sujeitos com SM têm mostrado que a causa da baixa concentração de HDL-colesterol é decorrente de uma elevação no seu catabolismo (20).

\section{Hipertensão arterial sistêmica (HAS)}

Vários estudos têm demonstrado a associação entre obesidade e hipertensão arterial e sua prevalência é 2,5 a 4,5 vezes maior em crianças e adolescentes obesos, e vários são os mecanismos que contribuem para essa hipertensão: ocorre uma disfunção do sistema nervoso simpático com aumento da frequência cardíaca basal e variabilidade da PA. A própria apneia do sono, frequente nos obesos, também contribui para o aumento do tônus simpático. A resistência à insulina tem papel na retenção renal de sal e aumenta a secreção de angiotensina II pelos adipócitos hipertrofiados. A angiotensina II interfere com a sinalização do receptor insulínico, inibindo a sua fosforilação à tirosina e estimulando a fosforilação em serina da subunidade beta e dos substratos 1 e 2 (IRS l e 2) (24,25). Em células vasculares endoteliais, a angiotensina II inibe a geração do óxido nítrico, contribuindo para vasoconstrição e hipertensão diastólica. No músculo cardíaco, ela inibe a captação da glicose e leva à hipertrofia miocárdica (7) e, nos rins, leva à ativação do sistema renina-angiotensina-aldosterona e induz à retenção de água, sódio e hipertensão sistólica (26). Ao lado disso, alterações vasculares estruturais, com disfunção endotelial e menor complacência vascular, também predispõem à HAS.

\section{Estado pró-inflamatório e pró-trombótico}

O excesso de tecido adiposo libera citocinas inflamatórias e, em resposta a esse estado inflamatório crônico, o organismo libera fatores pró-trombóticos. Portanto, no paciente obeso, é comum encontrarmos elevação dos seguintes marcadores: PCR, fator inibidor do ativador de plasminogênio (PAI-1) e fibrinogênio. Esses achados são mais comuns em pacientes com SM.

\section{Doença cardiovascular}

Indivíduos com SM têm seu risco cardiovascular aumentado e vários estudos têm demonstrado essa associação. O estudo de Botnia, que envolveu 4 mil adultos finlandeses e suíços, demonstrou que os pacientes com SM pelos critérios da Organização Mundial da Saúde (OMS) foram três vezes mais propensos a doença car- 
diovascular do que os que não tinham SM, e esta também foi associada a maior mortalidade por doença cardiovascular ( $12 \%$ versus $2 \%$ dos pacientes sem SM) (27).

\section{Diabetes melito tipo 2}

Concomitantemente à epidemia de obesidade infantil, a incidência e a prevalência de DM2 também se elevaram significativamente (21). Os estudos indicam que grande parte dos diabetes recém-diagnosticados em adolescentes é do tipo 2. Segundo a American Diabetes Association (ADA), $8 \%$ a $40 \%$ dos casos novos diagnosticados são diabetes não autoimunes. Estudos clínicos identificaram os principais fatores de risco associados ao DM2 juvenil: obesidade, antecedente familial para DM2, puberdade, certas etnias, alto ou baixo peso ao nascimento e presença de SM (21). Alguns fatores foram determinantes desse processo nas últimas décadas: alterações na dieta e redução da atividade física, além de exposição fetal à hiperglicemia em razão de diabete gestacional.

O DM2 aparece preferencialmente em adolescentes em fase puberal com idade média de 13 anos e afeta mais meninas do que meninos numa proporção de $1,5: 1$ a $3: 1$.

A ADA recomenda a realização de glicemia de jejum a cada dois anos em crianças com idade $>10$ anos ou em início de puberdade para triagem diagnóstica. Em 2004, a ADA descreveu situações de risco para o desenvolvimento de DM2: glicemia de jejum alterada (100 a $125 \mathrm{mg} / \mathrm{dL}$ ) e/ou intolerância à glicose (glicemia $2 \mathrm{~h}$ OGTT [teste de tolerância oral à glicose]: 140 a 200 $\mathrm{mg} / \mathrm{dL})(28)$.

\section{Esteatose hepática}

Cada vez mais fica evidente que o acúmulo de gordura no fígado, no paciente obeso, está longe de ser um evento sem consequências futuras. Tal situação é definida como doença hepática gordurosa não alcoólica (non alcoholic fatty liver disease - NAFLD) e abrange um espectro de alterações que vão desde a esteatose hepática assintomática, com aminotransferases normais ou pouco elevadas, até a esteato-hepatite (nonalcoholic steatohepatitis - NASH). Em crianças não obesas, a NAFLD afeta $2,6 \%$, mas, em obesos, chega a $77 \%$ e, em nossos pacientes do grupo de obesidade do ICr-HC-FMUSP, chega próximo a $25 \%$.

Não se conhece exatamente a história natural da NAFLD, mas a mortalidade em pacientes com esteato-hepatite é mais alta naqueles que apresentam fibrose ou inflamação hepática (29). O que é alarmante é que, no grupo de crianças e adolescentes obesos, a esteato-hepatite pode evoluir para cirrose hepática.

Tem sido demonstrada uma forte relação entre esteatose hepática e hiperinsulinemia/resistência à insulina. Sugere-se mesmo que a NAFLD pode ser considerada a manifestação hepática da SM e, nesse contexto, associa-se à obesidade, à resistência à insulina, à dislipidemia e ao metabolismo glicídico alterado.

Um modelo aceito para a patogênese do fígado gorduroso é a hipótese dos "dois eventos": a resistência à insulina compromete os depósitos de lípides e a lipólise em tecidos sensíveis à insulina, levando a um fluxo aumentado de ácidos graxos livres para o fígado, com acúmulo de triglicérides nos hepatócitos. O "segundo evento" é o estresse oxidativo, que ativa citocinas inflamatórias (como TNF alfa) e gera espécies reativas de oxigênio, tais como radicais hidroxil e ânions superóxido. Tais substâncias reagem com o excesso de lípides e forma peróxidos. Os produtos lipídicos oxidados podem lesar as células, interferindo com a função de membrana ou estimulando a fibrose pelas células hepáticas estreladas (30).

Não fica claro se a esteatose hepática é consequência ou causa dos desarranjos metabólicos na síndrome metabólica, mas é evidente que a esteatose hepática representa uma preocupação maior em crianças e adolescentes obesos e deve ser pesquisada sistematicamente, para que se possam instituir medidas terapêuticas antes que lesões irreversíveis se estabeleçam.

Conclui-se que, apesar das dificuldades diagnósticas da SM em crianças e adolescentes, sabe-se que a prevalência dessa síndrome é muito superior em crianças obesas. Desse modo, quando estamos diante de uma criança obesa que começa a apresentar alterações metabólicas, há necessidade de intervenções precoces para evitar as complicações futuras, como DM2, esteatose hepática e doenças cardiovasculares, que podem surgir precocemente na fase mais produtiva do indivíduo, com repercussões no âmbito pessoal, social e econômico. O escopo do tratamento é a mudança no estilo de vida.

Declaração: os autores declaram não haver conflitos de interesse científico neste estudo.

\section{REFERÊNCIAS}

1. Nelson RA, Bremer AA. Insulin resistance and metabolic syndrome in the pediatric population. Metab Syndr Relat Disord. 2010;8(1):1-14.

2. Cruz ML, Goran MI. The metabolic syndrome in children and adolescents. Curr Diab Rep. 2004;4(1):53-62. 
3. Spicuzza L, Leonardi S, La Rosa M. Pediatric sleep apnea: early onset of the "syndrome"? Sleep Med Rev. 2009;13:111-22.

4. Fernandez JR, Reddent DT, Pietrobelli A, Allison DB. Waist circunference percentiles in nationally representative samples of African-American, European-American, and Mexican-American children and adolescents. J Pediatr. 2004:145(4):439-44.

5. Katzmarzyk PT. Waist circunference percentiles for canadian youth 11-18 of age. Eur J Clin Nutr. 2004;58(7):1011-5.

6. Maffeis C, Pietrobelli A, Grezzani A, Provera S, Tatol L. Waist circunference and cardiovascular risk factors in prepubertal children. Obes Res. 2001;9(3):179-87.

7. Carr DB, Utzasghneider $\mathrm{KM}$, Hull RL, Kodama K, Retzlaff BM, Brunzell JD, et al. Intra-abdominal fat is a major determinant of the National Cholesterol Education Program Adult Treatment Panel III criteria for the metabolic syndrome. Diabetes. 2004;53(8):2087-94.

8. Weiss R, Dziura J, Burgert TS, Tamborlane WV, Taksali SE, Yeckel $\mathrm{CW}$, et al. Obesity and the metabolic syndrome in children and adolescents. N Engl J Med. 2004;350(23): 2362-74.

9. Mokha JS, Srinivasan SR, DasMahapatra P, Fernandez C, Chen W, $\mathrm{Xu} \mathrm{J}$, et al. Utility of waist-to-height ratio in assessing the satatus of central obesity and related cardiometabolic risk profile among normal weight and overweight/obese children:The Bogalusa Heart Study. Pediatrics. 2010;10:73-9.

10. Fisberg M, Cintra TP, Costa RF, Santos LC. Obesidade infanto-juvenil: epidemiologia, diagnóstico, composição corporal e tratamento. In: Setian N, et al. Obesidade na criança e no adolescente. 1.ed. São Paulo: Roca; 2007. p. 7-28.

11. Instituto Brasileiro de Geografia e Estatística (IBGE). Análise da disponibilidade domiciliar de alimentos do estado nutricional no Brasil. Disponível em: www.ibge.com.br. Acesso em: Nov 24, 2006.

12. Ferreira $A P$, Oliveira $C E$, França NM. Metabolic syndrome and risk factors for cardiovascular disease in obese children: the relationship with insulin resistance (HOMA-IR). J Pediatr (Rio J). 2007;83(1):21-6.

13. Freedman DS, Khan LK, Dietz WH, Srinivasan SR, Berenson GS. Relationship of childhood obesity to coronary heart disease risk factors in adulthood: The Bogalusa Heart Study. Pediatrics. 2001;108(3):712-8.

14. Goodman E, Daniels SR, Morrison JA, Huang GB, Dolan LM. Contrasting prevalence of and demographic disparities in the World Health Organization and National Cholesterol Education Program Adult Treatment Panel III definitions of metabolic syndrome among adolescents. J Pediatr. 2004:145(4):445-51.

15. Duncan GE, Perri MG, Theriaque DW, Hutson AD, Eckel RH, Stacpoole PW. Exercise training, without weight loss, increases insulin sensitivity and postheparin plasm lipase activity on previously sedentary adults. Diabetes Care. 2003;26(3):557-62.

16. Goodman E, Whitaker RC. A prospective study of the role of depression in the development and persistence of adolescent obesity. Pediatrics. 2002;110(3):497-504.
17. World Health Organization. Definition, Diagnosis and Classification of Diabetes Mellitus and its Complications. Part 1: Diagnosis and Classification of Diabetes Mellitus. Geneva: Department of Noncommunicable Disease Surveillance; 1999. p. 31-3.

18. Anderson PJ, Critchley JA, Chan JC, Corkram CL, See ZS, Thomas $\mathrm{GN}$, et al. Factor analysis of the metabolic syndrome: obesity vs insulin resistence as the central abnormality. Int Obes Relat Metas Disord. 2001;25(12):1782-8.

19. Steinberger J, Daniels SR, Eckel RH, Hayman L, Lustig RH, McCrindle $\mathrm{B}$, et al. Progress and challenges in metabolic syndrome in children and adolescents: a scientific statement from the American Heart Association Atherosclerosis, Hypertension, and Obesity in the Young Committee of the Council on Cardiovascular Disease in the Young: Council on Cardiovascular Nursing; and Council on Nutrition, Physical Activity, and Metabolism. Circulation. 2009;119:628-47.

20. Griffin JE, Ojeda SE. Textbook of endocrine physiology. 5.ed. Oxford: Oxford University Press; 2004.

21. Center for Disease Control and Prevention (CDC). National Center for Health Statistics; 2000.

22. Lottenberg SA, Glezer A, Turatti LA. Metabolic syndrome: identifying the risk factors. J Pediatr (Rio J). 2007;83(5 Suppl):S204-8.

23. Franco RR, Fuks FB, Damiani D. Síndrome metabólica: prevenindo complicações a longo prazo. In: Damiani D. Endocrinologia na Prática Pediátrica. 2.ed. Barueri, SP: Manole; 2011. p. 70-87.

24. Saad MJ, Velloso LA, Carvalho CR. Angiotensin II induces tyrosine phosphorylation of insulin receptor substrate 1 and its association with hosphatidylinositol 3-kinase in rat heart. Biochem J. 1995;310(3):741-4.

25. Carvalheira JB, Calegari VC, Zecchin HG, Nadruz W Jr, Guimarães $\mathrm{RD}$, Ribeiro $E B$, et al. The cross-talk between angiotensin and insulin differentially affects phosphatidylinositol 3-kinase- and mitogen-activated protein kinase-mediated signaling in rat heart: implications for insulin resistance. Endocrinology. 2003; 144:5604-14.

26. Andreozzi F, Laratta E, Sciacqua A, Perticone F, Sesti G. Angiotensin II impairs the insulin signaling pathway promoting production of nitric oxide by inducing phosphorylation of insulin receptor substrate-1 on Ser312 and Ser616 in human umbilical vein endothelial cells. Circ Res. 2004;94:1211-8.

27. Scott M, Grundy MD, Bryan BJ, James L, Sidney C. Definition of metabolic syndrome: Report of the National Heart, lung, and blood institute/ American Heart Association conference on scientific issues related to definition. Circulation. 2004;109(3):433-8.

28. American Diabetes Association. Diagnosis and Classification of Diabetes Mellitus. Diabetes Care. 2004;27(Suppl1)55:S10.

29. Ekstedt M, Franzén LE, Mathiesen UL, Thorelius L, Holmqvist M, Bodeman G, et al. Long-term follow-up of patients with NAFLD and elevated liver enzymes. Hepatology. 2006;44:865-73.

30. Browning JD, Horton JD. Molecular mediators of hepatic steatosis and liver injury. J Clin Invest. 2004;114:147-52. 